\title{
análisis \\ de la influencia de algunas variables en la extracción y ensayo a compresión de probetas-testigo de hormigón
}

ADOLFO DELIBES LINIERS, ingeniero de caminos Jefe del Laboratorio de INTEMAC

\begin{abstract}
sinopsis En el presente artículo, el autor describe los ensayos realizados en INTEMAC con objeto de estudiar los procesos de extracción, conservación y ensayo de probetas-testigo de hormigón. Se analiza la influencia en los resultados de una serie de factores, con vistas al estudio de estructuras terminadas. Se hace referencia a los criterios que deben seguirse para el establecimiento

462-3 de un Plan de ensayos y para la interpretación de los resultados.
\end{abstract}

\section{INTRODUCCION}

El estudio de la resistencia del hormigón en estructuras acabadas es un problema que aún no está totalmente resuelto. Por un lado, los criterios de muestreo presentan cierta incertidumbre, pues resulta difícil decidir el número de muestras, los lugares de la estructura de los que deben tomarse, los tipos de ensayos a realizar, etc. De otra parte, la incertidumbre continúa planteándose, incluso en mayor grado, a la hora de interpretar los resultados obtenidos en estructuras que presentan cierta complejidad.

Los procedimientos de ensayo han sido, quiza, más estudiados que aquellas etapas anterior y posterior a ellos.

Para el estudio de la resistencia del hormigón en una estructura acabada se realizan unos ensayos que denominamos "de información". Pueden ser éstos destructivos o no. Cuando se combinan varios tipos de ensayo, el problema estriba en la necesidad de relacionar los resultados obtenidos de una u otra manera. En principio, es muy recomendable rechazar la práctica de tomar decisiones sobre la base exclusiva de resultados de ensayos no destructivos.

Son, por tanto, los "ensayos de información destructivos" los que orientan el criterio de quien haya de tomar una decisión en base a la calidad del hormigón en una estructura ya terminada. El ensayo destructivo más común es el ensayo a compresión de probetas-testigo de hormigón.

El trabajo más completo que conocemos sobre este tema es el publicado por N. Petersons en la revista RILEM de noviembre-diciembre de 1971 (2). En él se indican unos criterios para el establecimiento de los planes de muestreo (número de testigos, tamaño de los mismos, puntos de la estructura de los que deben extraerse, etc.) y de los métodos de ensayo (forma de las probetas, modo de conservación, relación de diámetro del testigo a tamaño máximo del árido, etc.). Los criterios de aceptación o rechazo son los que parecen más complicados de establecer de forma clara y sencilla, aunque hayan sido también estudiados. En este estudio se recogen gran cantidad de ensayos efectuados por diversos autores. Sobre esta base, se encuentran en redacción por un comité mixto - CEB/CIB/FIP/RILEM - unas especificaciones para el estudio de la resistencia del hormigón en estructuras acabadas.

En 1971 se llevaron a cabo en el Instituto Técnico de Materiales y Construcciones (INTEMAC) los ensayos que se describen en el presente trabajo. En ellos se intentaban estudiar las in- 
fluencias de algunas variables que afectan a un plan de extracción de testigos, y a los métodos de ensayo en los resultados finales, comparándolos con los obtenidos con probetas moldeadas, fabricadas con hormigón fresco.

Sólo cuando pueda establecerse una normativa adecuada para la fijación de estos aspectos. estaremos en condiciones de realizar el siguiente paso: establecer las directrices para el análisis de los resultados de ensayo, especificando los criterios de aceptación o rechazo de las estructuras en estudio.

\section{PLAN DE ENSAYOS}

Los ensayos hechos partieron de la fabricación de dos muros de hormigón armado de $0,35 \times 1,45 \mathrm{~m}$ de sección, hormigonados verticalmente. Se dispuso una armadura, simétrica en ambas caras, formada por barras $\varnothing 14$ de acero ordinario.

El objeto de los ensayos era estudiar la influencia, en los valores a determinar de la resistencia, de las siguientes variables:

a) Dirección de extracción del testigo en relación con la dirección del hormigonado.

b) Altura del muro a la que se extrae el testigo.

c) Tamaño de la probeta, conservando la relación normalizada de altura a diámetro $(\mathbf{h} / \varnothing=2)$.

d) Condiciones de conservación de los testigos desde su extracción hasta el momento del ensayo. (Se ensayaron testigos conservados a intemperie, con H.R. del 40 al $65 \%$, y otros sumergidos en agua durante las 48 horas previas a su ensayo).

e) Influencia de la proximidad de armaduras.

f) Influencia de la inclusión de armaduras, barra $\varnothing 14$, en dirección transversal al testigo.

\section{DESCRIPCION DE LOS ENSAYOS REALIZADOS}

En la figura 1 se representan una sección longitudinal y otra transversal del muro 1. En ella se indica la forma de extraer los testigos de dicho muro. Análogamente se representa el muro 2 en la figura 2. Finalmente, en la figura 3 se indica la referencia que se asignó a cada testigo.

Del muro 1 se extrajeron 48 testigos de $15 \mathrm{~cm}$ de diámetro y $35 \mathrm{~cm}$ de longitud, para el estudio de la influencia de la dirección de extracción y de su altura en la pieza.

Del muro 2 se extrajeron 24 testigos de 10 y $15 \mathrm{~cm}$ de diámetro, para el estudio del resto de las variables anteriormente enunciadas.

El hormigón fue suministrado de una central de hormigonado, empleándose cemento P-350 y árido de $35 \mathrm{~mm}$ de tamaño máximo.

Se hormigonaron los muros en posición vertical, compactándose por vibración.

Se realizaron 5 tomas de muestras de hormigón fresco, durante el vertido, para la fabricación de 30 probetas cilíndricas de $15 \times 30 \mathrm{~cm}$ compactadas por picado con barra o por vibración. Se conservaron a intemperie o en cámara, en condiciones normalizadas (H.R. $=95 \%$ y $20^{\circ} \mathrm{C}$ ), hasta su ensayo a la misma edad que los testigos. 

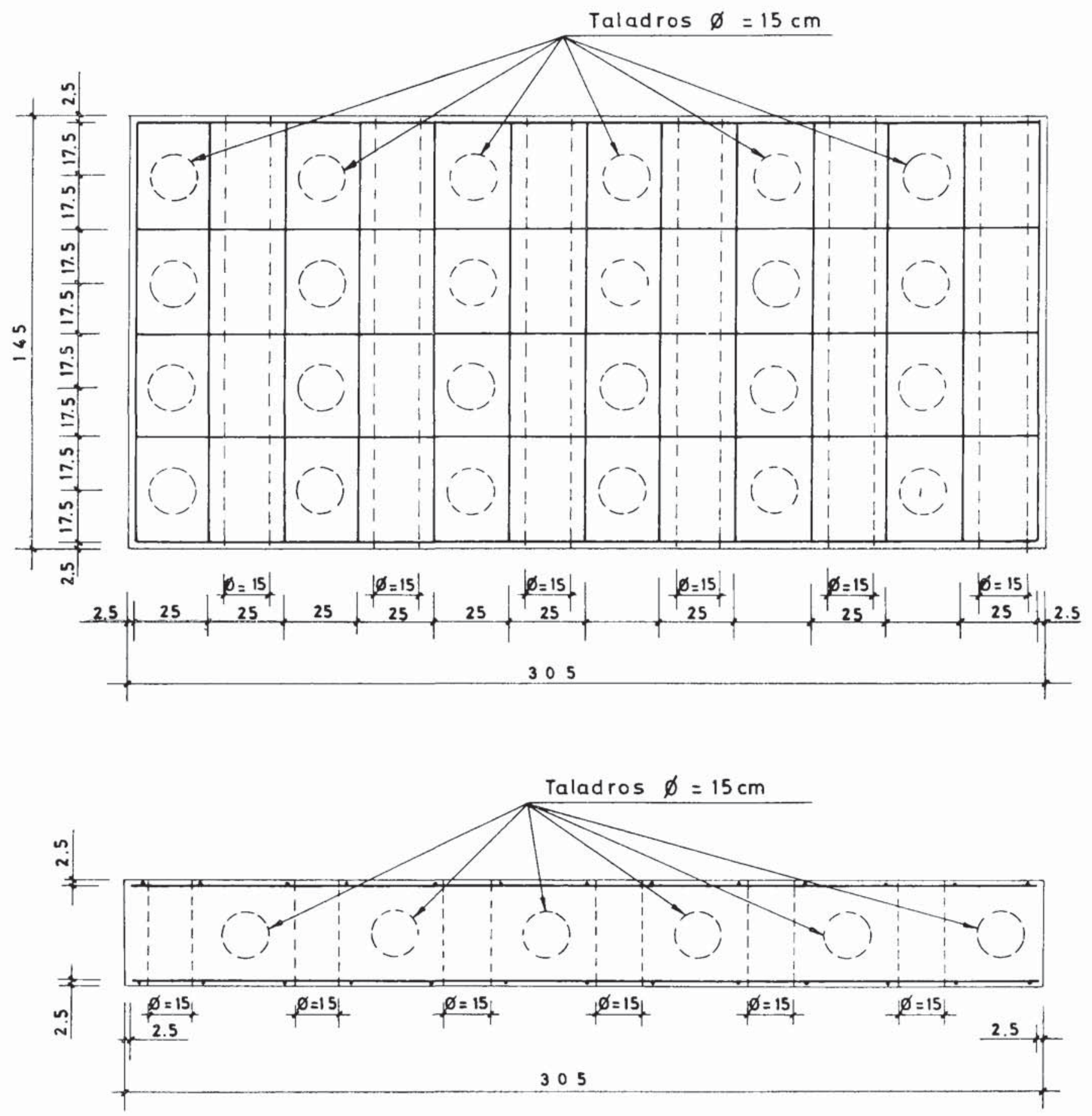

MURO 1. CARACTERISTICAS Y POSICION DE TALADROS

Fig. 1

Los testigos se extrajeron con sonda rotativa PIXIE-6, provista de coronas de diamante tipo Diaborit. Fueron tallados con sierra de disco de material análogo y diámetro suficiente para cortar toda la sección sin necesidad de mover el testigo. Los ensayos a compresión se hicieron en prensa Amsler de $100 \mathrm{t}$ de capacidad.

Se replanteó, previamente a la extracción, la posición de todas las barras que constituían la armadura, mediante sondeo magnético realizado con pachómetro.

Los trabajos de extracción de testigos dieron comienzo cuando el hormigón tenía 14 días de edad. 


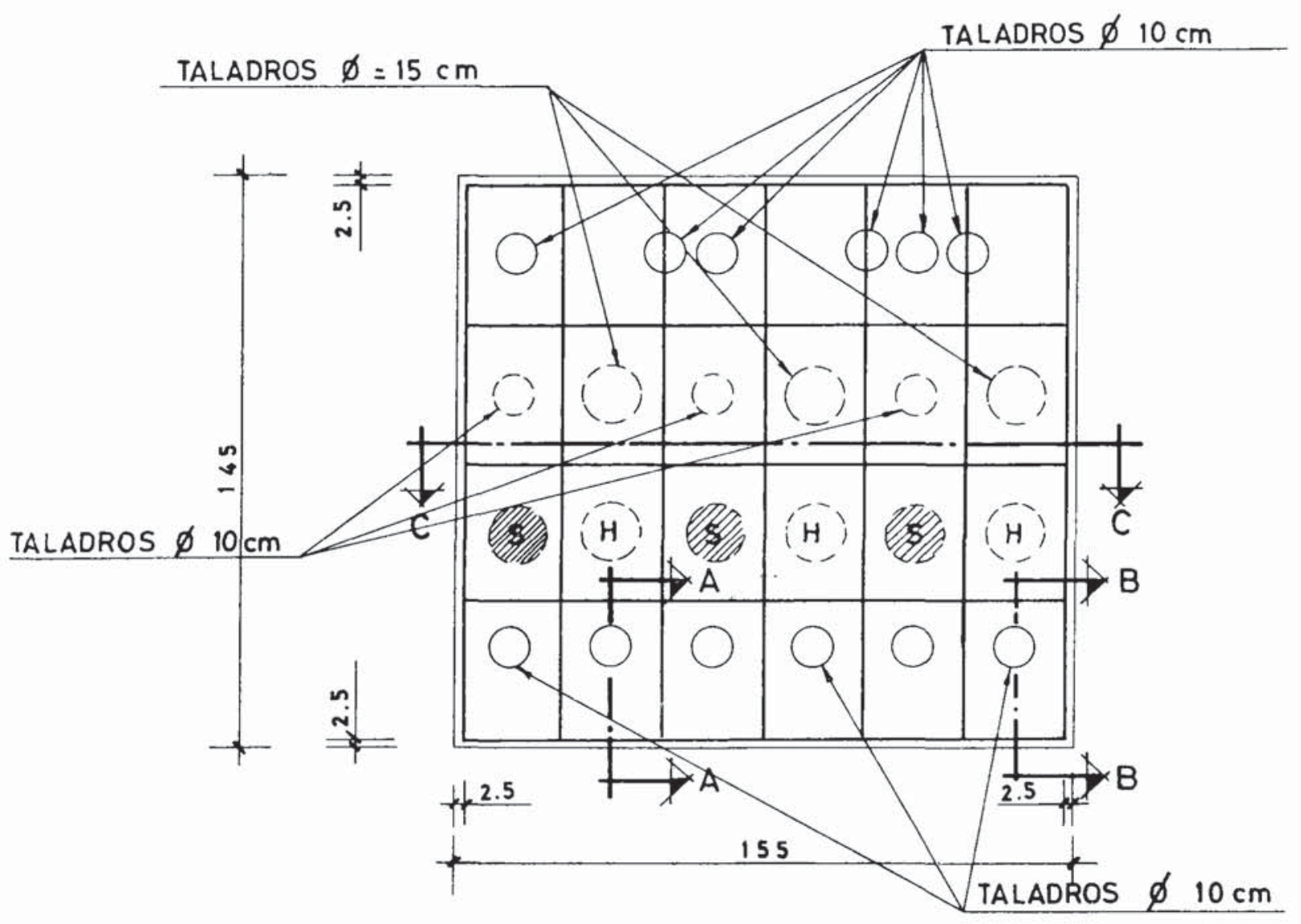

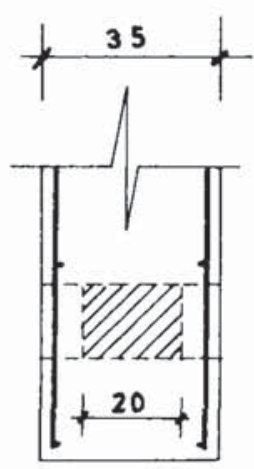

SECCION A-A

Testigo interior

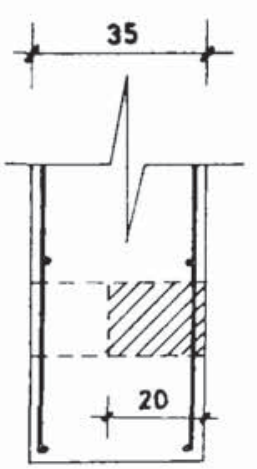

SECCION B-B

Testigo de borde
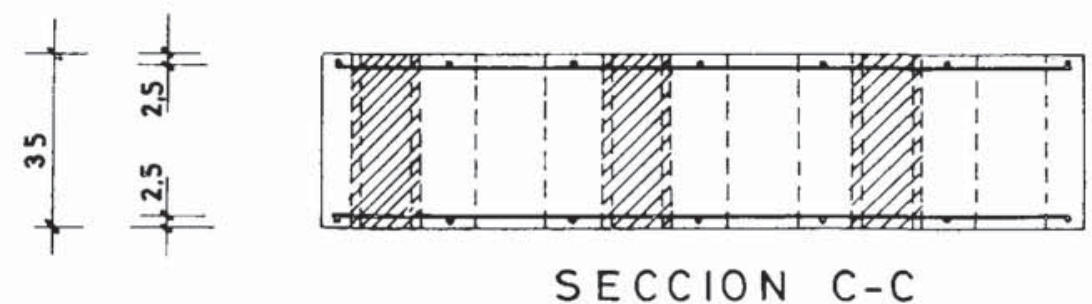

Fig 2

MURO 2. CARACTERISTICAS Y POSICION DE LOS TALADROS 


$$
\text { CODIGO: } \mid \begin{aligned}
& H-\text { Horizontal } \\
& \text { V- Vertical }
\end{aligned}
$$

MURO -1

\begin{tabular}{|c|c|c|c|c|c|c|c|c|c|c|c|}
\hline$(11 \mathrm{H})$ & $11 \mathrm{~V}$ & $12 \mathrm{H}$ & $12 \mathrm{~V}$ & $13 \mathrm{H}$ & $13 \mathrm{~V}$ & $(14 \mathrm{H}$ & $14 \mathrm{~V}$ & $(15 \mathrm{H})$ & $15 \mathrm{~V}$ & $(16 \mathrm{H})$ & $16 \mathrm{~V}$ \\
\hline$(21 \mathrm{H})$ & $21 \mathrm{~V}$ & $(22 \mathrm{H})$ & $22 \mathrm{~V}$ & $(23 \mathrm{H})$ & $23 \mathrm{~V}$ & $(24 \mathrm{H})$ & $24 \mathrm{~V}$ & $(25 \mathrm{H})$ & $25 \mathrm{~V}$ & $(26 \mathrm{H})$ & $26 \mathrm{~V}$ \\
\hline$(31 \mathrm{H})$ & $31 \mathrm{~V}$ & $(32 \mathrm{H})$ & $32 \mathrm{~V}$ & $(33 \mathrm{H})$ & $33 \mathrm{~V}$ & $(34 \mathrm{H}$ & $34 \mathrm{~V}$ & $(35 \mathrm{H})$ & $35 V$ & $(36 \mathrm{H})$ & $36 \mathrm{~V}$ \\
\hline $41 \mathrm{H}$ & $41 \mathrm{~V}$ & $(42 \mathrm{H})$ & $42 \mathrm{~V}$ & $(43 \mathrm{H})$ & $43 V$ & $4.4 \mathrm{H}$ & $44 \mathrm{~V}$ & $(45 \mathrm{H})$ & $45 \mathrm{~V}$ & $46 \mathrm{H}$ & $46 \mathrm{~V}$ \\
\hline
\end{tabular}

\section{MURO - 2}

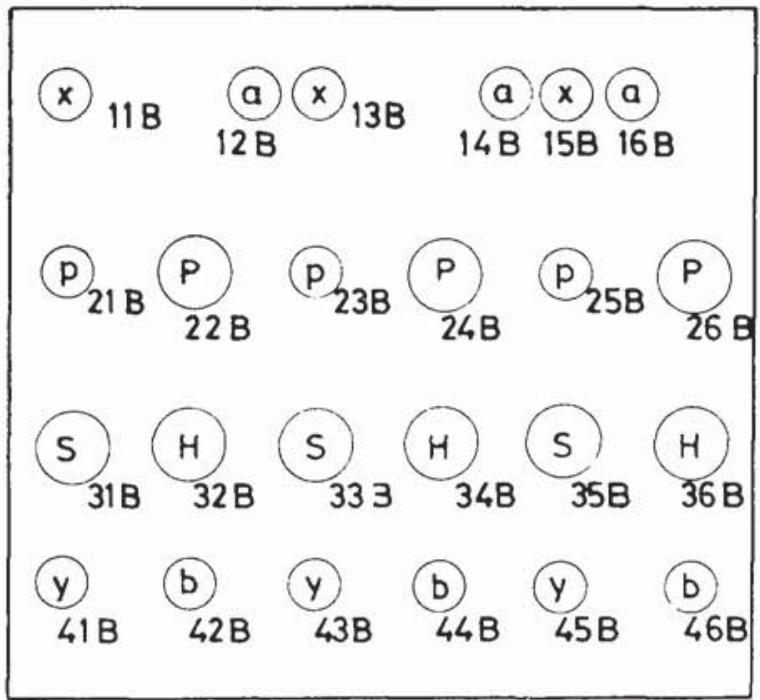

$$
\begin{aligned}
& \text { CODIGO } \\
& a=\text { con armadura } \\
& x=\sin \text { armadura } \\
& \mathrm{P}=\varnothing=10 \mathrm{~cm} \\
& \mathrm{P}=\varnothing \mathrm{cm} \\
& \mathrm{S}=\text { seca } \\
& H=\text { saturada } \\
& b=\text { de borde } \\
& y=\text { interior }
\end{aligned}
$$

Fig. 3

\section{resumen y comentarios de los resultados del ensayo}

En los cuadros 1 y 2 se han resumido los resultados de ensayo obtenidos. A continuación los comentaremos, estudiando la influencia de cada una de las variables que se han hecho intervenir en el plan de ensayos. 
CUADRO 1. MURO 1. RESUMEN DE RESULTADOS $\left(\mathrm{kp} / \mathrm{cm}^{2}\right)$

\begin{tabular}{|c|c|c|c|c|c|c|c|c|c|c|c|c|}
\hline \multirow[t]{2}{*}{$\begin{array}{l}\text { VARIABLES EN ESTLOIO } \\
\text { Vease codigo en figura } 3\end{array}$} & \multicolumn{2}{|c|}{$\begin{array}{l}\text { Direccion } \\
\text { de } \\
\text { Extraccion }\end{array}$} & \multicolumn{5}{|c|}{$\begin{array}{l}\text { Paralela a la } \\
\text { dirección de } \\
\text { hormiganedo. (v) }\end{array}$} & \multicolumn{5}{|c|}{$\begin{array}{l}\text { Perpendicular a la } \\
\text { direccion de harmi } \\
\text { gonado. (H) }\end{array}$} \\
\hline & \multicolumn{2}{|c|}{$\begin{array}{c}\text { Novel } \\
\text { de } \\
\text { Extraccion }\end{array}$} & 1 & 2 & 3 & 4 & \multirow{7}{*}{ 趈 } & 1 & 2 & 3 & $\Delta$ & \multirow{7}{*}{ 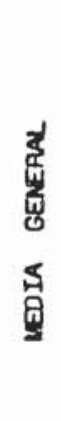 } \\
\hline & NQ de Probet & 1 & 243 & 223 & 279 & 251 & & 251 & 260 & - & 253 & \\
\hline & $n$ & 2 & 191 & 255 & 238 & 200 & & - & - & 237 & 220 & \\
\hline & n & 3 & 197 & 238 & - & - & & 263 & 220 & - & - & \\
\hline & 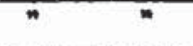 & 4 & 299 & 255 & - & 251 & & 229 & 247 & - & 250 & \\
\hline & $n$ & 5 & - & 251 & 274 & 235 & & 229 & 216 & 216 & 218 & \\
\hline & $"$ & 6 & 232 & - & 261 & 250 & & 237 & 227 & - & 229 & \\
\hline & MEDIA & & 215 & 244 & 275 & 251 & 246 & 242 & 234 & 226 & 234 & 234 \\
\hline & & + & 28 & 11 & 13 & 9 & - & 21 & 26 & 11 & 19 & - \\
\hline & U DOF ERSTUV & - & 49 & 21 & 14 & 16 & - & 13 & 18 & 10 & 16 & - \\
\hline & RECOARIDO ( & $\mathrm{p} / \mathrm{am}^{2}$ & 79 & 32 & 27 & 25 & - & 34 & 44 & 21 & 35 & - \\
\hline
\end{tabular}

La resistencia media de las probetas moldeadas es de $248 \mathrm{kp} / \mathrm{cm}^{2}$, con un recorrido (diferencia entre el resultado máximo y el mínimo) de $28 \mathrm{kp} / \mathrm{cm}^{2}$.

CUADRO 2. MURO 2. RESUMEN DE RESULTADOS

\begin{tabular}{|c|c|c|c|c|c|c|c|c|}
\hline $\begin{array}{c}\text { VARIABLES } \\
\text { EN } \\
\text { ESTUOIO }\end{array}$ & $\begin{array}{l}\text { ALTERNATIVA } \\
\text { vase codigo en } \\
\text { figura } 3\end{array}$ & \multicolumn{3}{|c|}{$\begin{array}{l}\text { RESULTADOS } \\
\left.(\mathrm{kp} / \mathrm{cm})^{2}\right)\end{array}$} & $\begin{array}{l}\text { MEDIAS } \\
\left(\mathrm{kp} / \mathrm{cm}^{2}\right)\end{array}$ & $\begin{array}{l}\text { DISPEASION } \\
\text { RESPECTO A LA } \\
\text { MEDIA (kp/cm²) }\end{array}$ & $\begin{array}{l}\text { RECORRIDO } \\
\left(\mathrm{kp} / \mathrm{cm}^{2}\right)\end{array}$ & OBSERVACIONES \\
\hline \multirow[b]{2}{*}{ ARMADUATR } & $x$ & 251 & 294 & 191 & 219 & $\begin{array}{l}+32 \\
-28\end{array}$ & 60 & $\begin{array}{l}\text { Incluye barra } \\
\text { Testigos } \varnothing 10 \mathrm{~cm}\end{array}$ \\
\hline & a & 227 & 211 & 214 & 217 & $\begin{array}{l}+10 \\
-\quad 6\end{array}$ & 16 & $\begin{array}{l}\text { No 1ncluye berra } \\
\text { Testigos } \emptyset 10 \mathrm{~cm}\end{array}$ \\
\hline \multirow{2}{*}{ DIAMETRO } & 15 & 214 & 185 & 192 & 197 & $\begin{array}{l}+17 \\
-12\end{array}$ & 29 & Standard \\
\hline & 10 & 201 & 207 & 201 & 203 & $\begin{array}{l}+4 \\
-2\end{array}$ & 6 & - \\
\hline \multirow{2}{*}{ HUMEDAD } & $\mathrm{H}$ & 185 & 215 & 209 & 203 & $\begin{array}{l}+12 \\
-13\end{array}$ & 30 & Stander \\
\hline & $\mathrm{s}$ & 259 & 253 & 226 & 246 & $\begin{array}{l}+13 \\
-20\end{array}$ & 33 & Ensayada seca \\
\hline \multirow{2}{*}{ CONPACTACION } & $B$ & 136 & 209 & 229 & 208 & $\begin{array}{l}+21 \\
-22\end{array}$ & 43 & $\begin{array}{l}\text { Incluye paramento } \\
\text { Testigas } 10 \mathrm{~cm}\end{array}$ \\
\hline & $Y$ & - & 210 & 201 & 205 & - & 9 & $\begin{array}{l}\text { Interior. } \\
\text { Testigos } 10 \mathrm{~cm}\end{array}$ \\
\hline
\end{tabular}

La resistencia media de las probetas moldeadas es de $252 \mathrm{kp} / \mathrm{cm}^{2}$, con un recorrido (diferencia entre el resultado máximo y el mínimo) de $18 \mathrm{kp} / \mathrm{cm}^{2}$. 
Debe añadirse que las resistencias de probetas moldeadas compactadas en las mismas condiciones que los muros (vibradas) y las picadas con barra dan lugar a resultados en que no se apreció variación alguna. Ello fue motivo de considerar globalmente todos estos ensayos.

\section{Influencia de la dirección de extracción del testigo}

Se obtiene una resistencia media un $5 \%$ inferior, en testigos extraídos perpendicularmente a la dirección de hormigonado, a la de testigos extraídos paralelamente a ella (fig. 24.1). La dispersión de los resultados es mayor, sin embargo, en estos últimos. En ello puede influir la forma de efectuar la extracción, que es más dificultosa.

Los testigos con mayores dispersiones, extraídos en la dirección del hormigonado, se cortaron con la sonda trabajando en posición horizontal, con lo que su trabajo se dificulta.

\section{INFLUENCIA DE LA ALTURA DE EXTRACCION}

(Se indica para cada nivel el resultado mínimo, el medio y el máximo obtenidos).

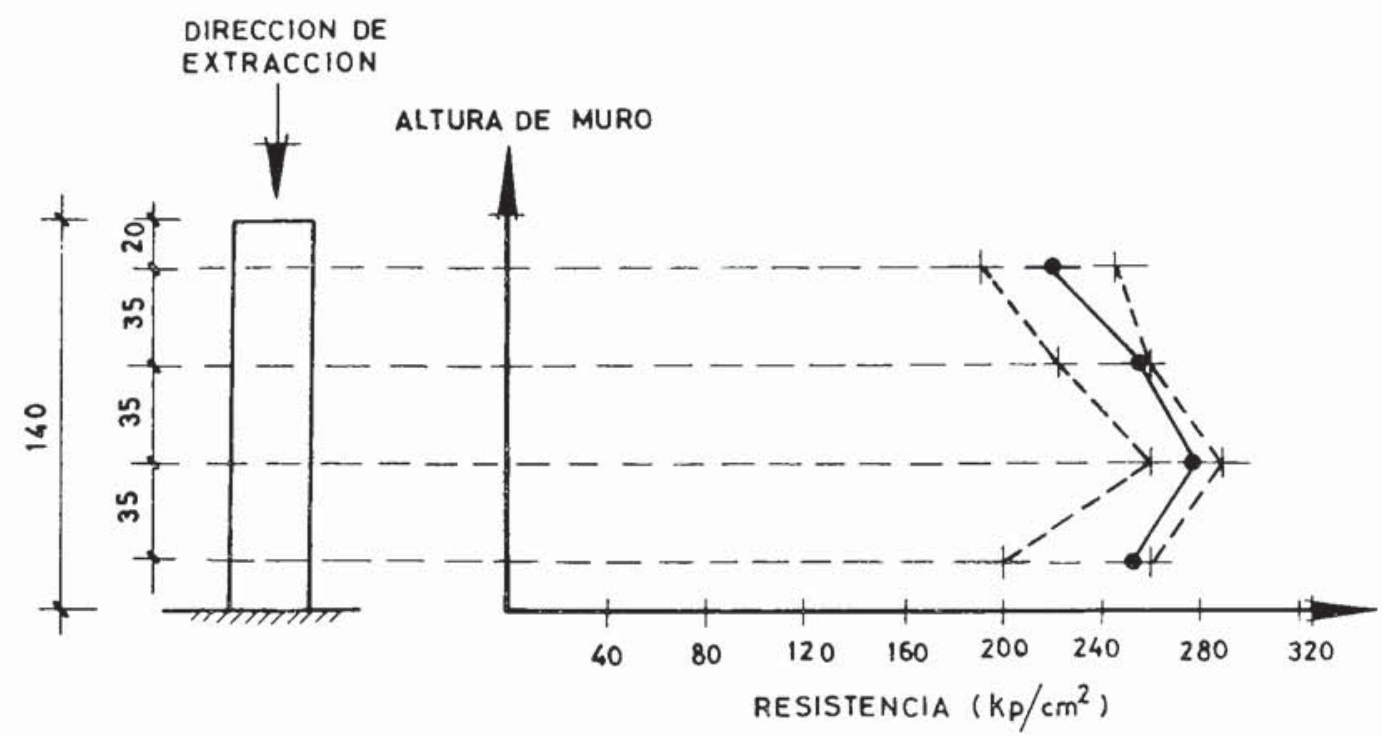

ALTURA DE MURO

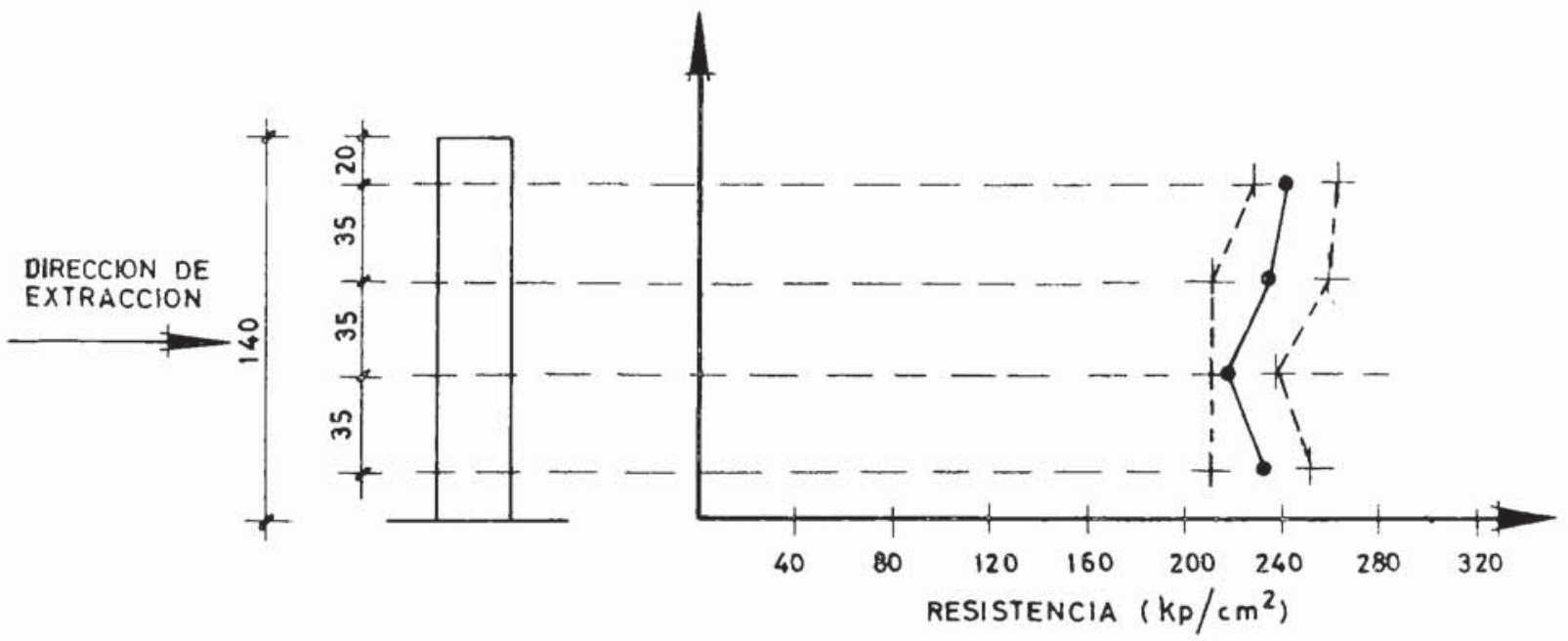

Fig. 4.1 
Otros estudios llegan a conclusiones análogas (1) y (5), o bien indican diferencias inapreciables (2) y (3), camufladas por las dispersiones de ensayo.

Evidentemente, lo usual es extraer los testigos perpendicularmente a la dirección del hormigonado (en pilares no queda otra solución). En consecuencia obtendremos resultados que, en cualquier caso, nos van a dejar del lado de la seguridad si no les aplicamos ningún tipo de corrección.

\section{Influencia de la altura de extracción del testigo}

En la figura 4.2 se muestran los resultados obtenidos con los testigos extraídos del muro 1 a diferentes alturas.

En los gráficos indicados puede observarse que la altura de extracción sólo influye apreciablemente en los resultados obtenidos con probetas extraídas en la dirección del hormigonado.

INFLUENCIA DE LA DIRECCION DE EXTRACCION MURO 1
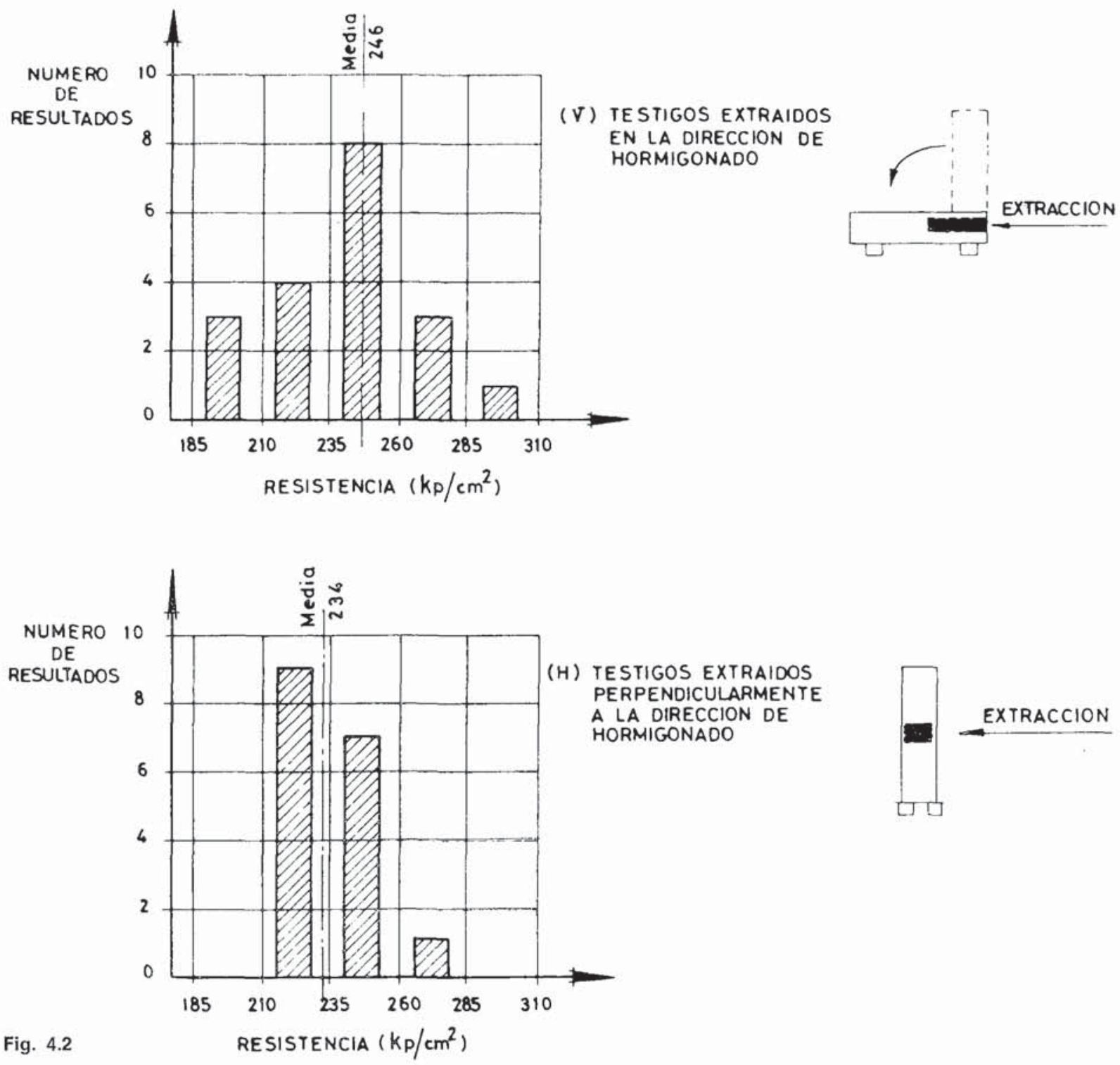
Ello es lógico si se piensa que a los testigos restantes no les afecta la parte más alta de la pieza. Los resultados vienen camuflados por el hecho de existir defectos de compacidad en la parte inferior de las piezas.

Petersons (2) Ilega a la conclusión, tras el análisis de resultados de todos los ensayos que recoge, de que la resistencia en piezas verticales es mínima en la inmediata vecindad de la cara superior del elemento y crece con la profundidad bajo dicha cara. Manifiesta que las diferencias observables dependen de las condiciones de curado y de la resistencia del hormigón, creciendo con ésta.

Bloem (5) encontró que las diferencias pueden ser del 20 al $40 \%$ entre probetas extraídas a $50 \mathrm{~cm}$ de profundidad y las más próximas a la superficie (10 a $15 \mathrm{~cm}$ de profundidad) en pilares.

Petersons (1) halló incrementos similares al descender hasta $50 \mathrm{~cm}$ en piezas análogas, manteniéndose la resistencia para mayores profundidades. Sin embargo, se observa una dispersión mayor en profundidad, que en algunos casos hace coincidir los resultados mínimos, aunque varíe la profundidad.

Meininger (4) deduce incrementos también dispersos (entre un 5 y un $25 \%$ ) al aumentar ligeramente la profundidad de extracción.

Viest, Elstner y Hognestad (21), al estudiar el comportamiento de soportes de hormigón armado que se habían vertido horizontalmente, llegaron a una capacidad del hormigón igual a su resistencia en probeta cilíndrica, mientras que si el hormigonado era vertical, dicha capacidad se veía reducida en un $15 \%$ aproximadamente (20). Este efecto se achacó a la causa que más adelante se indica.

De todo ello puede deducirse claramente una disminución real de la resistencia en las partes más altas de elementos hormigonados verticalmente. Este descenso, en piezas correctamente ejecutadas, deja de existir a unos $40 \mathrm{~cm}$ de la superficie en que se finalizó el hormigonado. Las distintas especificaciones tienen en cuenta esa disminución de la resistencia, afectando de un coeficiente de minoración 0,90 al valor de la resistencia en elementos hormigonados verticalmente.

Ha de tenerse en cuenta, sin embargo, que ello ocurría en piezas correctamente ejecutadas, como se ha indicado. Para hormigones excesivamente blandos el efecto puede ser mayor, afectando cualquier anomalía a las conclusiones que hemos establecido. Como es sabido, el descenso de resistencia estudiado es consecuencia de la mayor relación agua/cemento del hormigón superior, que recibe el agua que asciende de capas inferiores.

A estos efectos debe tenerse presente que el hormigón del muro 1 tenía una consistencia media de $5 \mathrm{~cm}$ determinada con cono de Abrams.

\section{Influencia de la inclusión de barras transversales de acero en la probeta-testigo}

En los pilares ensayados no se acusa la influencia en el resultado medio de la inclusión de una barra $\varnothing 14 \mathrm{~mm}$, transversalmente a la dirección de la carga de ensayo o eje longitudinal de la probeta-testigo. Sin embargo, se aprecia una mayor dispersión de los resultados cuando el testigo incluye armadura.

Lewandowski (6) ha publicado resultados de ensayos efectuados con probetas que incluian armadura (hasta un $3,4 \%$ de cuantía geométrica), observándose en ellos una disminución general no superior al $4 \%$ de la resistencia obtenida con probetas sin armadura. Gaynor (7) hizo ensayos análogos con probetas moldeadas, observando una influencia ligeramente mayor, que se traducía en un descenso de la resistencia.

En resumen, de acuerdo con Petersons (2), se puede concluir que normalmente la inclusión de armaduras transversales no tendrá efectos apreciables, o bien nos dejará ligeramente del lado de la seguridad, al ensayar a compresión las probetas-testigo. 
En cualquier caso, y respecto al tamaño del trozo de acero embebido, habrá que tomar las mismas medidas que las especificaciones aplican para el tamaño máximo del árido. La limitación del diámetro mínimo a 3 veces dicho tamaño máximo del árido llevaría a poder incluir barras $\varnothing 32$ en probetas de $10 \mathrm{~cm}$ de diámetro.

\section{Influencia de la proximidad de armaduras}

No se aprecia influencia de la proximidad de armaduras a los taladros. Sin embargo, es de esperar que en elementos estructurales deficientemente compactados, con barras gruesas o gran cantidad de armaduras, los testigos puedan resultar afectados por defectos locales de compactación.

\section{Influencia del diámetro del testigo}

El hormigón empleado tenía árido de tamaño máximo $35 \mathrm{~mm}$, entendido como aquel que supera al $85 \%$ de las partículas de árido.

En los ensayos realizados se obtiene una resistencia superior en un $3 \%$, con testigos $\varnothing 10$, que con los $\varnothing 15 \mathrm{~cm}$ e, incluso, menor dispersión. Si incluimos también en la comparación los testigos designados a $(\varnothing 10 \mathrm{~cm})$ y los designados $\mathbf{H}(\varnothing 15 \mathrm{~cm})$-que son comparables- observamos que las diferencias se mantienen y siguen siendo menos dispersos los resultados con $\varnothing 10 \mathrm{~cm}$ (véase la fig. 4.3).

\section{COMPARACION DE RESULTAdOS MEDIOS Y DISPERSIONES SEGUN EL DIAMETRO DEL TESTIGO}

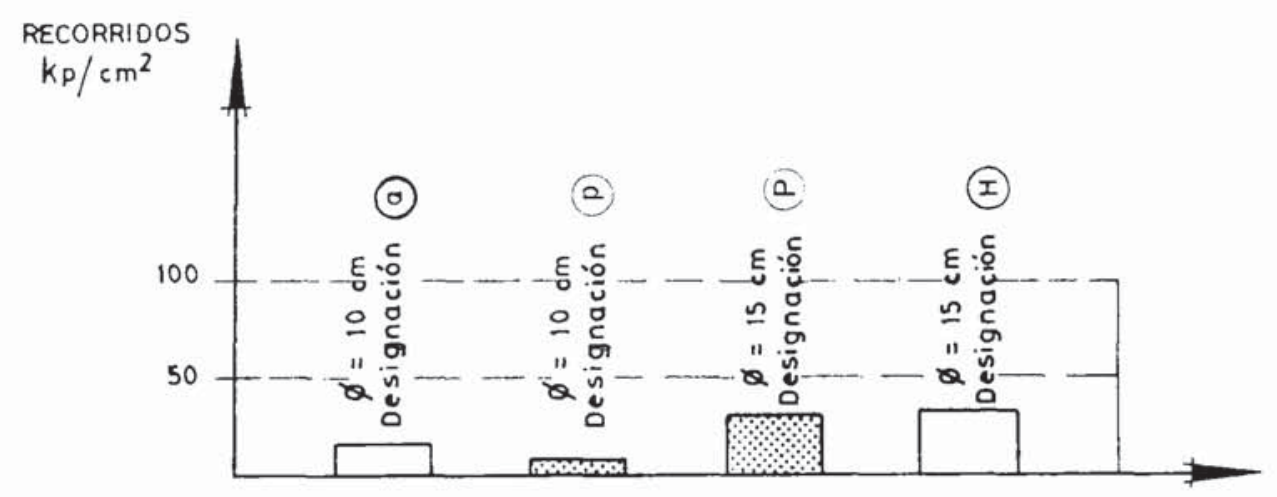

Fig. 4.3

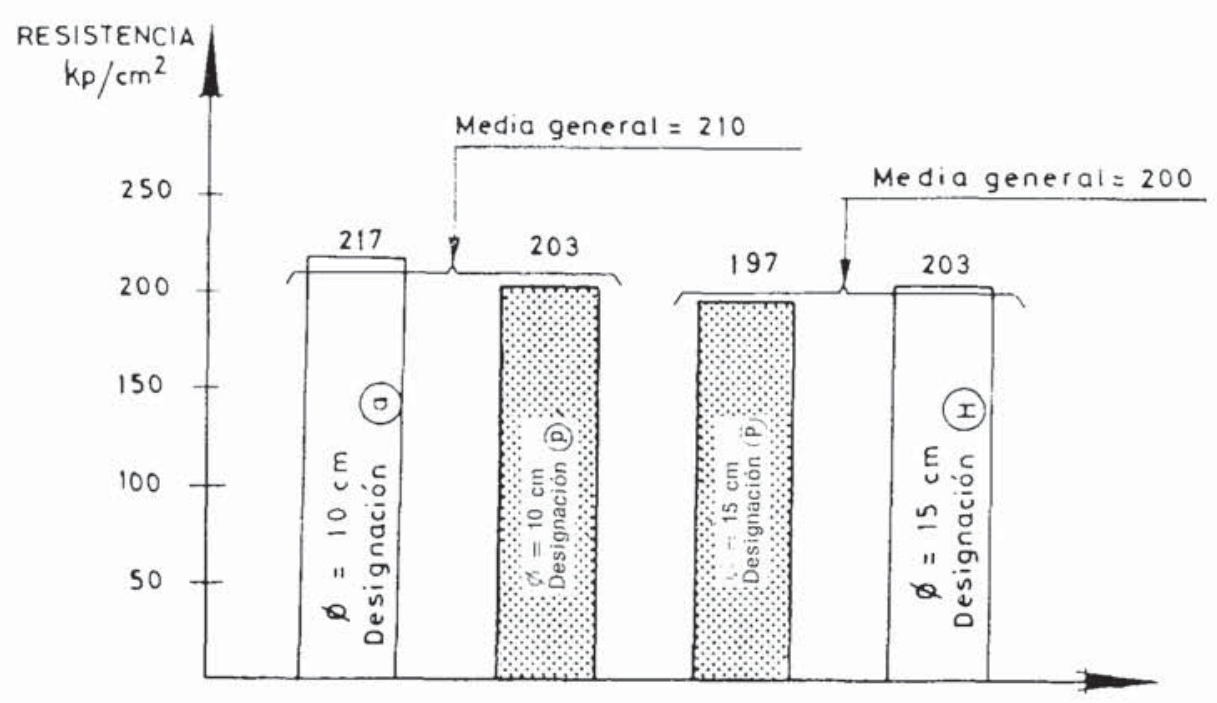


Según Neville (8) el efecto del tamaño de la probeta sobre su resistencia es consecuencia de un conjunto de factores: tamaño del árido, efecto pared, influencia de la falta de homogeneidad en las probetas mayores, etc. Señala, sin embargo, que en los casos usuales de tipos de hormigón y tamaños de probetas no existe un efecto apreciable y cita los resultados de ensayos de Plank y McNamara, ya en 1935, indicando que la resistencia obtenida con probetas de $7,5 \mathrm{~cm} \varnothing$ superaba en un $6 \%$ a la registrada en probetas standard $\varnothing 15 \mathrm{~cm}$. En cualquier caso, recomienda aumentar el número de ensayos si se reduce el tamaño de probeta.

Las limitaciones de distintas especificaciones, contemplando el tamaño máximo del árido, limitan a 3 ó 4 veces dicho tamaño, al diámetro mínimo de las probetas moldeadas.

Meininger (4) llega a la conclusión, empleando árido de $19 \mathrm{~mm}$ (3/4"), de que se obtienen resultados análogos con testigos de 5,10 y $15 \mathrm{~cm}$ de diámetro.

Campbell (9), sin embargo, acusa descensos de resistencia en probeta-testigo $\varnothing 10$ respecto a los testigos $\varnothing 15 \mathrm{~cm}$ con árido de machaqueo de $25 \mathrm{~mm}$.

No obstante, de acusarse influencia del diámetro, debe ser en sentido contrario al apuntado por Campbell, como indicaba Neville. Ello lo confirman los resultados de ensayo de W. Kerber (10), Kesler (11), Henzel y Freitag (12) y Petersons (1). Las diferencias encontradas no superan un $6 \%$ para diámetros de probeta entre 5 y $15 \mathrm{~cm}$.

La mayor parte de las investigaciones citadas muestran además que la disminución del diámetro de testigo lleva a un aumento en la dispersión, lo que hace aconsejable un mayor número de testigos.

Existen también (13) referencias de investigaciones que confirman la de Meininger, con testigos de 7,5 a $15 \mathrm{~cm}$ de diámetro y $25 \mathrm{~mm}$ de tamaño máximo del árido.

Por tanto, los resultados de nuestros ensayos confirman los obtenidos en otras investigaciones, indicando que existe una disminución de la resistencia con el aumento del tamaño de probeta. En todo caso, las probetas pequeñas deben tener un diámetro superior a 3 veces el tamaño máximo del árido, siendo conveniente tener en cuenta que puede producirse un aumento en la dispersión, aunque no lo acusen nuestros ensayos ("), que verdaderamente son pocos en número.

En la práctica lo más útil y cómodo será el empleo de testigos $\varnothing 10 \mathrm{~cm}$, como ponen de manifiesto también algunos de los estudios que hemos citado (2) y (3) y permiten las especificaciones (14), (15) y (16).

\section{Influencia del estado de saturación de las probetas en el momento del ensayo}

Aunque los ensayos llevados a cabo son muy pocos para cuantificar la influencia del estado de saturación de la probeta, sí resulta clara la importancia de este aspecto. Se han ensayado probetas-testigo conservadas a intemperie sin otro tratamiento que su refrentado, paralelamente a otras que se han mantenido sumergidas en agua de 40 a 48 horas.

Los resultados obtenidos en probeta saturada son superados por los de las secas (la humedad ambiente oscilaba entre el 45 y $60 \%$ ) en un $20 \%$.

Lo anterior se ve confirmado en realidad por todos los ensayos hechos con testigos extraídos del muro 2. La influencia de las restantes variables queda amortiguada por esta otra. Así la

["] Téngase presente que las sondas utilizadas para la extracción de testigo trabajan mucho más holgadamente cuando se extraen de $10 \mathrm{~cm}$ de diámetro. Ello conduce a la posibilidad de que aparezcan mayores dispersiones con testigos grandes, por haberles podido afectar el proceso de extracción con una máquina trabajando al límite de sus posibilidades. 
resistencia media de las probetas directamente ensayadas fue de $246 \mathrm{kp} / \mathrm{cm}^{2}$, frente a los $209 \mathrm{kp} / \mathrm{cm}^{2}$ de las sumergidas en agua previamente al ensayo, es decir, se mantiene la diferencia del $20 \%$ al considerar la totalidad de las probetas procedentes del muro 2.

Neville, en su "Properties of Concrete", ya citado, señala que el efecto del estado de saturación depende del proceso seguido (saturar una probeta seca o secar una saturada), del tamaño de probeta y de la velocidad a que se produce el cambio, además de las características propias del hormigón. En general, con probetas, indica que su resistencia aumenta al disminuir el grado de saturación. Cuantificar este efecto es difícil, citándose los ensayos de Buteher en 1958, que registró incrementos entre el 5 y $10^{\circ} \%$ con hormigón de $350 \mathrm{kp} / \mathrm{cm}^{2}$. En todo ello debe tenerse en cuenta que puede alterarse el resultado en hormigones mal curados, ávidos de agua, en que puede darse un aumento de resistencia cuando se les proporciona ese agua que necesitan y se ensayan al cabo de un cierto tiempo.

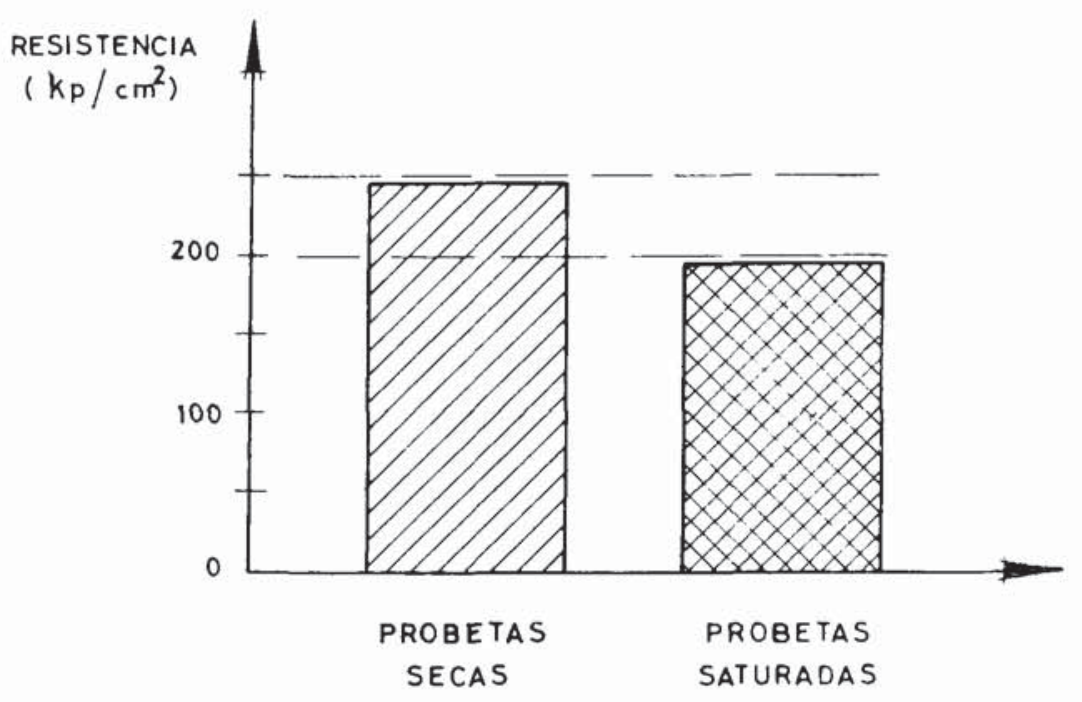

Bloem, ya citado, dice que manteniendo los testigos 7 días al aire tras su extracción se aumenta su resistencia un $20 \%$ respecto de los testigos sumergidos durante 48 horas. Ello con diferentes procesos de curado y diámetros de testigo entre 5 y $15 \mathrm{~cm}$. Estos resultados concuerdan exactamente con los nuestros. Quizá haya que añadir a lo anterior que la edad de ensayo era de 90 días.

Meininger muestra que la resistencia disminuye por inmersión en agua y también Petersons confirma los resultados obtenidos y la cuantía de la influencia de este factor, que estima entre un 15 y un $20 \%$.

Algunas especificaciones exigen la saturación de los testigos una vez tallados y previamente a su ensayo (ASTM, UNE, GBV). Otras, en cambio, permiten que se conserven en ambiente con humedad media o escasa, menor del $60 \%$, durante un período mínimo de 2 a 3 días o de 7 días en otros casos. Así, la norma ACl 301-1971 señala que las probetas-testigo pueden conservarse al aire, en ambiente del laboratorio, durante 7 días, antes de su ensayo. Realmente, el hormigón de una estructura normal, en servicio, es difícil que esté saturado, y así lo entiende también Petersons en sus "Recomendaciones". El único motivo que nos llevaría a saturar los testigos, reduciendo con ello su resistencia, seria lograr una mejor correlación con los ensayos normalizados sobre probetas moldeadas, ya que éstas, en el momento del ensayo, se encuentran saturadas. 


\section{Correlación de los resultados con los obtenidos en probetas moldeadas}

Como se ha indicado, se ensayaron probetas moldeadas procedentes de diferentes tomas de muestra de hormigón fresco y que fueron compactadas por picado con barra (Norma UNE 7240 ) o por vibración. Los resultados con ambas fueron análogos.

Las resistencias medias de las probetas moldeadas y de los testigos se comparan en el cuadro siguiente:

\begin{tabular}{c|c|c}
\hline \multirow{2}{*}{ MURO } & \multicolumn{2}{|c}{$\mathbf{f}_{\mathrm{c}}$ media en $\mathrm{kp} / \mathrm{cm}^{2}$} \\
\cline { 2 - 3 } & \multicolumn{2}{|c}{ PROBETAS } \\
\cline { 2 - 3 } 1 & MOLDEADAS & TESTIGO \\
\hline 2 & 248 & 240 \\
& 252 & $207^{*}$ \\
\hline
\end{tabular}

* No se tienen en cuenta los ensayos sobre testigos no saturados.

Observamos una mayor resistencia en las probetas moldeadas. También se obtuvieron con ellas, como era de esperar, menores dispersiones.

La publicación del M.O.P., "Resistencia Característica y Control de Calidad" de septiembre de 1972 (18), recomienda interpretar los resultados de ensayo, cuando ha de establecerse un valor característico de la resistencia para compararlo con el valor de especificación o de proyecto, utilizando lo que denomina "estimador A". Este estimador de la resistencia se aplica afectando de un determinado coeficiente al mínimo resultado obtenido. Dicho coeficiente es función del coeficiente de variación; es decir, de la uniformidad del hormigón y del número de ensayos efectuados. (Es éste uno de los estimadores adoptados por la Instrucción Española E.H.-73). Además indicaba aquella publicación que se dividiese a los resultados obtenidos por un coeficiente 0,9 por tratarse de testigos extraídos de elementos que no se ejecutan tan cuidadosamente como las probetas moldeadas de control. En nuestro caso el proceso de cálculo citado condujo a los resultados siguientes:

\begin{tabular}{c|c|c}
\hline \multirow{3}{*}{ MURO } & \multicolumn{2}{|c}{$\mathbf{f}_{\mathrm{ck}}$ estimada en $\mathrm{kp} / \mathrm{cm}^{2}$} \\
\cline { 2 - 3 } & \multicolumn{2}{|c}{ PROBETAS } \\
\cline { 2 - 3 } & MOLDEADAS & TESTIGO \\
\hline 1 & 229 & 221 \\
2 & 224 & 211 \\
\hline
\end{tabular}

Valga lo expuesto como un ejemplo que expresa la validez del criterio indicado. No se pretende dar normas de aplicación general para la interpretación de resultados de un estudio de la resistencia del hormigón en una estructura acabada, como ya indicábamos en la introducción al presente trabajo. 


\section{conclusiones}

Los ensayos llevados a cabo por el Instituto Técnico de Materiales y Construcciones (INTEMAC) en 1971, que concuerdan con la mayor parte de los estudios que hemos relacionado y, por otro lado, se vieron confirmados por las "Recomendaciones" de Petersons (2), llevan a las siguientes conclusiones:

a) Existe una cierta influencia de la dirección de extracción de testigos en relación con la de hormigonado. Sin embargo, será pequeña y del lado de la seguridad en los casos usuales.

b) En zonas muy próximas a la cara superior de elementos hormigonados verticalmente, la resistencia del hormigón es menor. En estudios prácticos de estructuras terminadas podría adoptarse la regla de que en los $50 \mathrm{~cm}$ superiores la resistencia del hormigón será como máximo el $90 \%$ de la del resto del elemento.

c) Se pueden utilizar los diámetros usuales de testigo siempre que superen el triple del tamaño máximo del árido. Conviene tener en cuenta el posible aumento de las dispersiones al emplear diámetros pequeños. Ello puede subsanarse aumentando el número de ensayos.

d) No se ha apreciado influencia alguna de la proximidad de armaduras ni de su inclusión transversal a las probetas-testigo. Generalmente podrá despreciarse su efecto.

e) Es muy notable la influencia del estado de saturación de la probeta en el momento del ensayo. Las probetas saturadas dan lugar a resistencias un $20 \%$ inferiores a las conservadas al aire, en ambiente normal de laboratorio. Puede seguirse la norma $\mathrm{ACl} 301$ y ensayar las probetas sin someterlas a una saturación previa y permitiendo su secado durante un cierto período en un ambiente normalmente seco.

\section{bibliografía}

1. PETERSONS, N.: "Strength of Concrete in Finished Structures". Swedish Cement and Concrete Institute 1964. Reprint 26.

2. PETERSONS, N.: "Recommendations for Estimation of Quality of Concrete in Finished Structures». Swedish Cement and Concrete Research Institute. No. 7014. 1971.

3. J. L. RAMIREZ ORTIZ y J. M. BARCENA DIAZ: "Aportación en torno a la valoración de la calidad resistente del hormigón de estructuras mediante probetas-testigo extraídas por corte». Art. 462-2. Informes de la Construcción. Instituto Eduardo Torroja. Madrid, 1974.

4. MEININGER, R. C.: "Effect of Core Diameter on Measured Concrete Strength". Journal of Materials, 3 1968, 2.

5. BLOEM, D. L.: "Concrete Strength Measurement», Cores Vs. Cylinders. ASTM Proceedings, Vol. 65, 1965.

6. LEWANDOWSKI, R.: “Einfluss von Bewehrungsabschnen». Betonstein-Zeitung, 1970.

7. GAYNOR, R. D.: "Effect of Horizontal Reinforcing Steel on the Strength of Molded Cylinders". Journal of the A.C.I., 62, 1965.

8. NEVILLE: "Properties of Concrete». Pitman Publishers, 1963. 
9. CAMPBELL y TOBIN: "Core and Cylinder Strengths of Natural and Light-weight Concrete». ACl Journal, Title n.․ 64-18, abril 1967.

10. WULFF KERBER: “Cilindros Vs. Corazones". México D.F. Septiembre-octubre 1969.

11. KESLER: "Effect of Length to Diameter Ratio on Compresive Strength». ASTM Proceedings, Vol. 59, 1959.

12. HENZEL y FREITAG: "Einflüsse auf die Ergebnisse bel Druckfestigkeitsprufüngen an Beton». Mitteilungen ans dem Institut für Massivban an der Technischen Hochschule Darmstadt. Helft 12, 1966.

13. M. LUISA DIAZ DE SMITTER: «Estimación de la calidad del Concreto en Estructuras acabadas. Medida de la resistencia del Concreto: Núcleo Vs. Cilindros». Boletín Técnico núm. 42 del IMME. Facultad de Ingeniería Caracas, abril-junio 1973.

14. PROPOSAL GBV: "Dutch Standard for Reinforced Concrete». 1971.

15. ASTM C-42: "American Standards for Testing Materials". 1964.

16. ACl-301-66: «Proposed revision». Junio 1971.

17. Normas UNE 7240,7241 y 7242 . Instituto Nacional de Racionalización. Madrid.

18. "Resistencia Característica y Control de Calidad". Comisión Permanente del Hormigón. M.O.P. Septiembre 1972.

19. Instrucción E.H.-73. Comisión Permanente del Hormigón. M.O.P. Octubre 1973.

20. HOGNESTAD, E.: «A study of Combined Bending and Axial Load in Reinforced Concrete Membersn. University of Illinois. Bulletin 399, Urbana, 1951.

21. VIEST, ELSTNER and HOGNESTAD: "Sustained Load Strength of Excentrically Loaded Columns". ACI Journal. Proc. Vol. 52, núm. 7, marzo 1956.

22. J. M. TOBIO: "Resistencia del hormigón en estructuras terminadas". Informes de la Construcción, núm. 203. Agosto-septiembre 1968.

\section{résumé}

Analyse de l'influence de quelques variables sur le prélèvement et I'essai à la compression de carottes de béton

Adolfo Delibes Liniers, ingénieur des Ponts et Chaussées

Dans cet article, l'auteur fait une descrip tion des essais réalisés en INTEMAC, dans le but d'étudier le nombre de carottes né. cessaires pour vérifier la résistance du béton dans les structures terminées et les normes les plus appropriées pour exécuter les essais d'information, destructifs ou non, définissant les critères d'acceptation ou de refus des structures objet d'étude.

\section{summary}

Analysis of the influence of some variables in the extraction and com. pression test of concrete test pieces

Adolfo Delibes Liniers, Civil engineer

This article describes the tests carried out at INTEMAC, with the aim of determining the number of test pieces that are necessary to prove the concrete strength in completed structures and the most appropriate norms to perform these tests-destructive or non. destructive, defining the acceptance or re. jection criteria for the structures under study.

\section{zusammenfassung}

Analyse des Einflusses einiger Variablen auf die Extraktion und Druckversuche an Betonprobestücke

Adolfo Delibes Liniers, Zivilingenieur

Dieser Artikel beschreibt die in INTEMAC ausgeführten Versuche, um zu untersuchen wie viele Probestücke notwendig sind, um die Festigkeit des Betons in fertigen Struk. turen festzustellen sowie die angebrachtes. ten Normen, um diese Informationsversuche auszuführen, zerstörend oder nicht zerstö. rend, und es werden die Annahme- und Zurückweisungskriteria der zu untersuchenden Strukturen definiert. 